Historic, archived document

Do not assume content reflects current scientific knowledge, policies, or practice 



\section{Fancher Creek Nurseries}

\section{SURPLUS LIST No. 1}

RUDOCRT: D

Fresno, Cal., February 24, 1906

The following is a list of stock which we have on hand, heeled in our packing grounds in the City of Fresno, the same being in first-class condition and ready for inmediate shipment. In addition to the list submitted, we will have a very fine stock of all the leading varieties of Olives, Oranges, Diciduous and Evergreen Ornamental Trees, as well as Palms and Shrubbery. We have an immense stock of CALIMYRNA FIGS, as well as other leading varieties, which can be dug on short notice.

This list is sent for the convenience of our customers, and orders will be filled for anything listed as long as the stock holds out. Address

GEO. C. ROEDING, Pres! and Manager

FANCHER CREEK NURSERIES,

Fresno, Cal.

\section{APPLE, ONE YEAR}

\section{ON APPLE}

A rkansas Black.

Baldwin ............... 440

Ben Davis.............. 430

Bismarck............. 320

Duchess of Oldenburg ...... 50

English Russett ...........

Esopus Spitzenburg..........

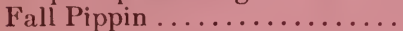

Fameuse.

Gano.................. 600

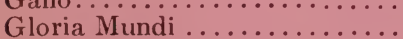

Glowing Coal ................

Golden Russett.............

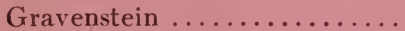

Hubbardston Nonsuch..........

Hyslop ................. I 20

Kentucky Redstreak........... 165

Keswick Codlin ..............

King of Tompkins County .....

Jonathan ...............

Lady Apple...............

Lawver.................

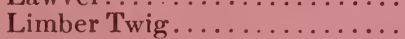

Mammoth Black Twig........

Maiden Blush..............

Marshall'sRed or RedBellflower

$3-4 \mathrm{ft}$.
500
330
400
190
20
50
70

400
25
20
80
200

30
40
50
300
300
20
80
100

ON APPLE

Missouri Pippin or Stone's Eu-

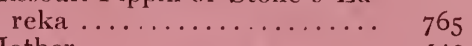
Mother................ 140

Nickajack .............. I40

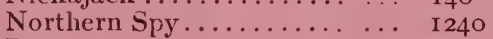

Paragon............... 140

Rambo............... $\quad 65$

Red Astrachan........... 400

Red June................. 200

Red Bietigheimer ........ ... 90

Red Pearmain ............ 340

Red Siberian $\ldots \ldots \ldots \ldots \ldots \ldots \ldots .20 \%$

Rhode Island Greening ....... I I I0o

Rome Beauty............ 90

Roxbury Russet............ I I35

Shockley ............. 70

Skinner's Pippin ............

Smith's Cider............. 870

Sonoma Seedling........... 50

Stark ............... 50

Swaar .....................

Transcendent ........... 135

White Winter Pearmain ..... 465

Wine Sap.

Yellow Bellflower........... Igo

Yellow Newton Pippin........ 200

Yellow Siberian........... 210
3-4 ft.

335

400

60

250

90

100

250

80

430

60

955

IOO

190

40

\section{PEAR, ONE YEAR}

\section{ON PEAR}

B. S. Fox...

Beurre Bosc ........... 90

Beurre Clairgeau....... .

Beurre d'Anjou ........ 30

Beurre Giffard ......... 170

Beurre Hardy

Bloodgood

Clapp's Favorite ........

Col. Wilder. ......... 60

Dana's Hovey ......... 210

Doyenne d'Alencon.......

Doyenne d'Ete......... 50

Doyenne du Comice .......

Duchesse d'Angouleme.. I60

Easter Beurre

Flemish Beauty

$46 \mathrm{ft}$.
100
70
40
25
40
90
40

80
210
110
60
35
30
90
60

\section{ON PEAR}

Forelle or Trout Pear.... IOO

Howell

Kieffer's Hybrid

Lawson or Comet

Le Conte.

Madeleine

P. Barry

Seckel

Winter Bartlet

Winter Nelis

Winter Serkel

ON QUINCE

Bartlett

Beurre Hardy

70 Glou Morceau

45 Winter Bartlett

\section{$923 \star$} rioulturas 


\section{CHERRY, ONE YEAR}

\section{ON MAZZARD}

Belle d'Orleans ............ $6_{5}$

Belle Magnifique ........... 35

Bing ................... I370

Black Eagle ............... 95

Black Tartarian ............. I70

California Advance.......... I 10

Centennial................... I70

Chapman ................. 55

Early Richmond .......... I 20

Elton .................. I60

Gov. Wood ............... 65

Great Bigarreau ........... I 135

Lambert................ 60

Lewelling ................ 210

May Duke.............. 80
ON MAZZARD

4-6 ft. $\quad 3-4 \mathrm{ft}$.

Napoleon Bigarreau or Royal

Anne................... I51o I 560

380

Olivet ...

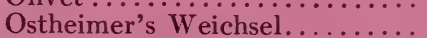

Reine Hortense .............

Rockport Bigarreau.........

60

Schmidt's Bigarreau......... 35

Windsor......................... 75

Yellow Spanish............ 50

ON MAHALEB ROOT ${ }_{4-6} \mathrm{ft}$

Bing $\ldots \ldots \ldots \ldots \ldots \ldots \ldots \ldots \ldots \ldots \ldots \ldots \ldots \ldots$

Early Richmond .......... r 105

Nay Duke .................
40

$3-4 \mathrm{ft}$.

\section{PLUM, ONE YEAR}

\begin{tabular}{|c|c|c|c|c|}
\hline & ON 1 & ACH & ON MYR & BOLAN \\
\hline & $4-6 \mathrm{ft}$. & $3-4 \mathrm{ft}$. & 4-6 ft. & $3-4 \mathrm{ft}$. \\
\hline$\cdots$ & 60 & & & \\
\hline (1, & 40 & & & \\
\hline Bartlett & & 45 & & \\
\hline Bavay's Green Gage . . . . & 30 & 40 & & \\
\hline Bradshaw & 55 & & 35 & \\
\hline Burbank ........ & 85 & & 160 & 40 \\
\hline Chalco & & & 45 & \\
\hline Cherry Plum ............... & & & 70 & \\
\hline Climax & I 40 & 70 & & \\
\hline Coe's Late Red. . . . . & & & So & 30 \\
\hline Columbia & & & IO5 & 20 \\
\hline Duane's Purple_. . . . . . . . & & & IO5 & \\
\hline $\begin{array}{l}\text { Early Golden Drop } \\
\text { General Hand }\end{array}$ & & & & \\
\hline $\begin{array}{l}\text { General }{ }^{\prime} \\
\text { Giant }\end{array}$ & 40 & I05 & 25 & \\
\hline Duke & & & 330 & \\
\hline Green Gage . . . & & & 20 & 60 \\
\hline ial Gage & 30 & & & \\
\hline 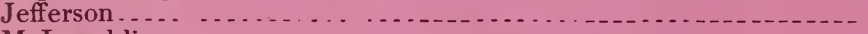 & & & 30 & IOO \\
\hline McLaughlin & & & 40 & \\
\hline Peach Plum & 20 & 30 & & \\
\hline Egg & So & & I 50 & \\
\hline ma & & & 60 & \\
\hline Shropshire Damson & 50 & & 9Io & I 50 \\
\hline Victoria & & & IOO & 20 \\
\hline n & & & I 70 & \\
\hline on $\ldots \ldots \ldots$. & 40 & & 25 & \\
\hline $\begin{array}{l}\text { Wild Goose } \text { We }_{-} \text {- } \\
\text { Yellow Egg. }\end{array}$ & & & $T=0$ & \\
\hline 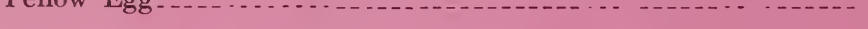 & & & 150 & 230 \\
\hline
\end{tabular}

\section{PRUNE, ONE YEAR}

\begin{tabular}{|c|c|c|c|c|c|c|c|c|c|}
\hline & \multicolumn{3}{|c|}{ ON ALMOND } & \multicolumn{3}{|c|}{ ON PEACH } & \multicolumn{3}{|c|}{ ON MYROBOLAN } \\
\hline & $6-\mathrm{st}$. & $4-6 \mathrm{ft}$. & $3-4 \mathrm{ft}$. & $6-8 \mathrm{ft}$ & $4-6 \mathrm{ft}$. & $3-4 \mathrm{ft}$. & $6-8 \mathrm{ft}$. & $4-6 \mathrm{ft}$. & $3-4 \mathrm{ft}$. \\
\hline $\begin{array}{l}\text { Fellenberg -...... } \\
\text { French }\end{array}$ & 1170 & I I $5^{\circ}$ & 200 & & 70 & 210 & 3360 & $\begin{array}{r}45 \\
1170\end{array}$ & $\begin{array}{r}80 \\
270\end{array}$ \\
\hline German & & & & & & & & 50 & 50 \\
\hline Hungarian ......... & 200 & & & 310 & & 50 & I Io & 50 & \\
\hline Imperial Epineuse.... & & 990 & 370 & & & & & 710 & 290 \\
\hline Robe de Sargent_.... & & & & & & & & 900 & 86́ \\
\hline Silver & 140 & $2 u$ & & & 140 & 20 & & 60 & \\
\hline $\begin{array}{l}\text { Sugar } \ldots \ldots \\
\text { Tragedy } \ldots \ldots \ldots\end{array}$ & 670 & 8Io & 380 & & I 80 & & 1830 & I 240 & I 340 \\
\hline
\end{tabular}




\section{GRAPES}

Alicante Bouschet

Almeria

Black Cornichon ...............

Black Ferrara ................

Black Hamburg ......

Black Malvoise -.............

Black Morocco ...................

Black Portugal

Black Prince.

Blue Spanish

Bowood Muscat .. .............

Burger

Brighton ........................

Cabernet Sauvignon ..............

Carignan

Catawba_....................

Cinsaut

Champion

Charbono .....................

Concord Cornichon White

Cornichon White

Delaware.......................

Early Madeleine .............

Early Ohio

rly Ohio - -

Feher Zagos -....................

Flame Tokay

Folle Blanche

Golden Chasselas_............

Gray Riesling . . . . . . . . . .

Grenache.........................

Goethe Gros Colman . .

Gros Colman...........................

Gros Marco . . . . .

Johannisberg Riesling ...........

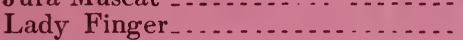

Malaga_-_._.

Mataro

Mission -... . . .

Mondeuse .........................

Moore's Diamond ........

Moore's Early ..............

Moselle Riesling .................

Mrs. Pince's Muscat _._._._._.

Muscat

Muscat Befere .................

Muscat Hamburg -..............

Mascat Rose .........

Niagara - .

Palomino Blanco

Pedro Ximenes

Petit Bouschet

Petit Syrah._.............

Pierce_-_._...................

Purple Damascus_...

Rose d'Italie . . . . . . . . . . . .

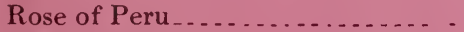

Sabal Kanski

Sauvignon Vert.

Semillon

Sultana

Thompson's Seedless

Triumph

Triumph

Trousseau

Ugni Blanc

Victoria Chassel

White Corinth

White Sweetwater or Chasselas de

Fontainbleau ...

Worden _ . . . .

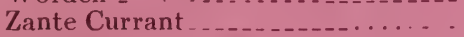

Zinfande 1

\section{PHYLLOXERA RESISTANT}

\section{VINES}

Aramon x Rupestris, Ganzin No. 1 Aestivalis x Colicola - R i p a ri a $\mathrm{x}$

Rupestris 554.5 -...-..-

Riparia Glorie de Montpellier

Riparia Glorie de Montpellier ....-

Riparia x Rupestris IOI-I4_........

Rupestris St. George ..........

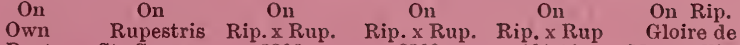

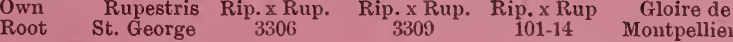

$\begin{array}{lllll}I 0000 & 3000 & \mathrm{I} I 00 & 430 & 250\end{array}$

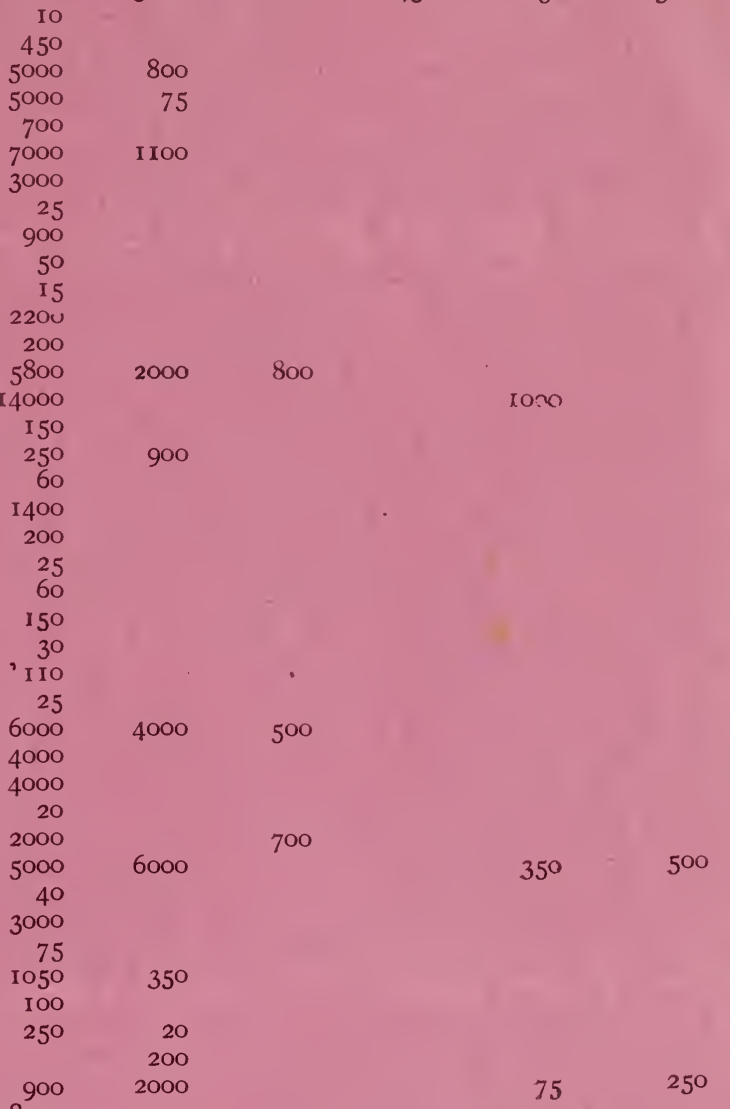

8000

1000

700

IOO

1000

$40 \quad$ I $500 \quad 3000$

60

50

25

125

2100

4000

7000

I000

600

75

I 1400

I 700

800

40
500

500

20

4000

200

100

2500

IOO

200
10000

$5^{\circ}$

600
3000

2500

275

400

600

200

20

5000

I 000

1000

I 5000

20000

I0000

20000 


\section{ROSES}

\section{STANDARD OR TREE ROSES}

Black Prinice

Climbing Kaiserin Augusta Victoria .... 6

Crimson Rambler _................ 22

Kaiserin .Aug. Victoria_........... 35

Maman Cochet_._....

Marechal Neil ................. 6

Perle des Jardins . . . . . . . . . . . . . . 50

Reine Marie Henrietta

Snowflake ..... - _.

Souvenir de Malmaison ............. . .

Sunset

White Maman Cochet. ............

\section{ROSES-GENERAL COLLECTION}

Admiral Dewey

American Bear

400

Antoine Mouton........ IOO

Banksian White _.................. 50

Beauty of Stapleford _.... 20

Belle Siebrecht _._._._-_._. 90

Black Prince . . . . . . . . . . . . . 45

Bridesmaid ...

Capt. Christy .......... IOO

Celine Forrestier................. 30

Charles Lefebre .... .............. 40

Cherokee Double.................. 20

Cheshunt Hybrid.-- 40

Climbing Bridesmaid................ 65

Climbing Capt. Christy ........... 45

Climbing Devoniensis _................ II 5

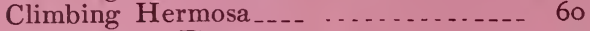

Climbing La France_ . . . _......... 25

Climbing Meteor................. 60

Climbing Niphetos _..._.......... 25

Climbing Perle des Jardins _..._._..... IOO

Climbing White Pet_._._._._.... 60

Climbing Wooton _................ 70

Clothilde Soupert _._._._._._..... 40

Crimson Rambler _........ 45

Duchesse de Brabant.............. 30

Duchess of Albany ........... 70

Duchess of Edinburgh _._._._._. 25

Empress of China ...

Empereur du Maroc._._._._._._. 40

Empress of India

Enchantress_....................... 40

Etoile de Lyon.................. I55

General Jacqueminot.............. 80

Gruss an Teplitz _....... I IO

Helen Gould .. _...... 45

Hermosa
Homer .......... 25

Hon. Edith Gifford $\ldots \ldots \ldots$

Jean Pernet............ . . . . _...... 30

Kaiserin August Victoria ...

La France

Lamarque .......... IIO

La Rosier or Prince Camille de Rohan _. 45

Louis Van Houtte ................... 35

Mabel Morrison .................. 20

Maman Cochet . _........

Mme. Alf. Carriere..... ............. 40

Mme. A. Veysset $\quad . . . . \ldots \ldots$ r.

Mme. C. Testout_..._....... I75

Mme. Chas, Wood ................. 90

Ime. Cusin

Mme. de Watteville................. 30

Mme. M. Kuppenheim.... . . . . . . . .. 30

Mme, Welche . $\quad 30$

Marchioness of Londonderry ........ 30

Marechal Nell _.............. 20

Margaret Dickson ............. 30

Marie Bauman ..................... 30

Merville de Lyon _... . . .

Meteor

Mignonette ..................... 50

Mrs. Degraw ........ $\quad . . . \ldots . . . .30$

Mrs. Pierpont Morgan.............. 30

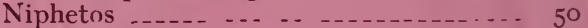

Papa Gontier.... 30

Paul Neyron_..._._._. ... . .... . . 50

Perfection des Blanches _._._._._..... 15

Persian Yellow.... $\quad . . . . . . . . . .6$

Pink Soupert .... ................. 20

Prairie Queen ....................... I5

Queen of Edgerly..... 40

Reine Marie Henriette............. 35

Reine Ogla de Wurtemburg ........... I5

Rubens ....................... 20

Salamander...

Senateur McNaughton .. $\quad \ldots . . . \quad$ IO

Snowflake ................ 50

Sourenir de President Carnot ... .... 40

Souvenir de Victor Hugo_............ . I5

Souvenir de Wooton_............... I5

Sunset _................... 40

Ulrich Brunner.

Valle de Chamounix _.............. 20

Vick's Caprice................. I5

White Baroness .................. 50

White Bath...

Williams Evergreen

W. A. Richardson .............. 30

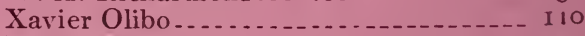

Yellow Rambler 